VIII ${ }^{\text {èmes }}$ Journées Nationales Génie Civil - Génie Côtier, Compiègne, 7-9 septembre 2004

\title{
Evolution d'une embouchure tidale en réponse au comblement de la baie associée: la baie de Marennes-Oléron et le Pertuis de Maumusson (Littoral Atlantique, France).
}

\author{
Xavier Bertin $^{(\mathrm{a})}$, Eric Chaumillon ${ }^{(\mathrm{b})}$, Aldo Sottolichio ${ }^{(\mathrm{c})}$ \\ ${ }^{(a)}$ Doctorant, ${ }^{(b)}$ Maître de conférence, Centre Littoral de Géophysique, \\ Université de La Rochelle, Avenue Michel Crépeau, \\ 17042 La Rochelle cedex1, France ; xavier.bertin@univ-Ir.fr \\ ${ }^{\circ}$ Maître de conférence, Département de géologie et océanographie, \\ UMR CNRS 5805-Epoc, \\ université Bordeaux-1, av. des Facultés, 33405 Talence cedex, \\ France; a.sottolichio@geocean.u-bordeaux.fr
}

\section{Résumé:}

La zone d'étude, située au milieu de la côte Atlantique, est constituée d'une baie tidale, la baie de Marennes-Oléron et de son embouchure, le pertuis de Maumusson. Des données bathymétriques fiables ont permis de montrer un comblement à la fois de la baie et de l'embouchure associée depuis 1824. L'étude des sections mouillées, les mesures de courants et les simulations hydrodynamiques mettent en évidence une diminution des prismes tidaux à Maumusson de $40 \%$. La diminution des débits tidaux dans la baie a été attribuée à son comblement d'une part et à une diminution conséquente de la propagation de la marée d'autre part. En accord avec la loi d'O'Brien [9] [10], cette diminution des prismes tidaux a, à son tour, entraîné un comblement de l'embouchure, dont les répercussions s'étendent jusqu'aux littoraux adjacents.

\section{Abstract:}

The study area is located in the middle of the Atlantic coast and include a tidal bay, Marennes-Oléron Bay, and its associated inlet, Maumusson Inlet. Accurate bathymetric data have permitted to evidence a filling in sediment of both the inlet and the tidal bay since 1824 . Cross sectional areas, current measurements and hydrodynamic modelling have revealed a decrease in tidal prisms in the order of $40 \%$ since 1824 at Maumusson Inlet. This decrease has been attributed to the filling in sediment of the bay and to a subsequent decrease in tidal wave propagation. According to O'Briens law [9] [10], this tidal prism decrease has led to a strong shoaling at the inlet, the repercussions extended up to adjacent shorelines.

Mots clés: Embouchure tidale, Prisme tidal, section mouillée, comblement sédimentaire.

\section{Introduction:}

Depuis les dernières décennies, l'importance économique et environnementale des systèmes embouchures tidales/lagunes ne cesse de croître [6]. La gestion de ces environnements fait face à de nouvelles préoccupations, comme le renouvellement en eau de la lagune pour l'aquaculture ou la stabilité des littoraux adjacents. Une bonne connaissance des relations entre les évolutions de la baie tidale et celles de l'embouchure apparaît donc essentielle. Probablement en raison de la nouveauté de ces préoccupations et de la difficulté à quantifier les évolutions morphologiques qui affectent les hauts d'estran de la lagune, peu d'études quantitatives ont été menées en ce sens. 
La Baie de Marennes-Oléron (Charente Maritime, France) n'échappe pas à ce constat puisqu'elle constitue le premier domaine ostréicole en Europe tandis que les littoraux adjacents à l'embouchure sont parmi les plus fréquentés de France.

\section{Zone d'étude:}

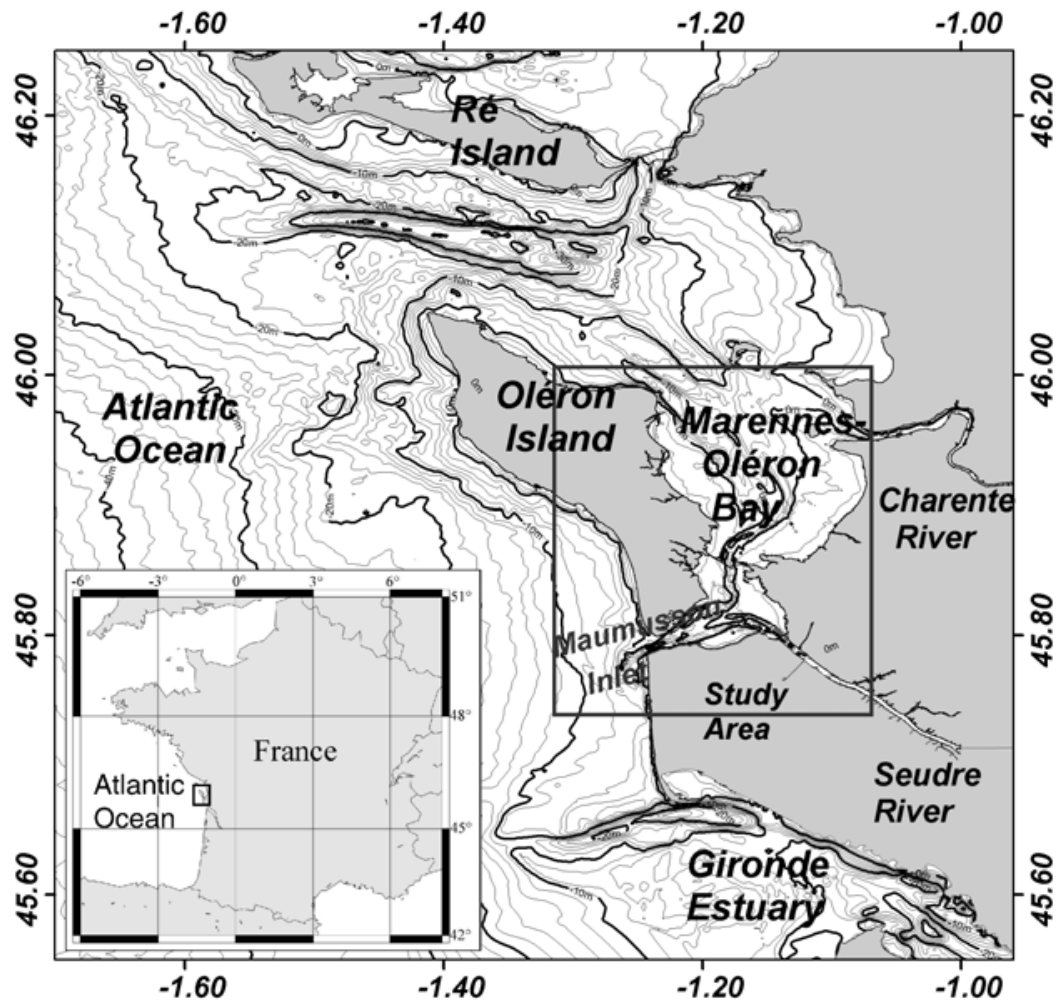

Figure 1. Localisation de la zone d'étude. Les traits gras correspondent aux isobathes $10 \mathrm{~m}$.

La Baie de Marennes-Oléron est une baie tidale de $150 \mathrm{~km}^{2}$, située au milieu de la côte Atlantique Française. Elle possède deux connections avec l'océan Atlantique: l'une au nord par le pertuis d'Antioche et l'autre au sud par l'embouchure tidale de Maumusson (Figure 1). Les domaines intertidaux représentent $60 \%$ de cette baie et constituent pour la plupart de large estrans vaseux. Deux petits fleuves se jettent dans la baie de Marennes-Oléron : la Charente au nord et la Seudre au sud. Les apports en eau continentale de ces deux fleuves sont toutefois très faibles face aux volumes d'eau mis en jeux par la marée.

La marée qui affecte la zone d'étude est de type semi-diurne et varie de moins de $2 \mathrm{~m}$ en morte-eaux à plus de $6 \mathrm{~m}$ en vive-eaux. Les courants associés sont faibles et rotatifs au large, mais importants dans les chenaux de l'embouchure tidale où ils atteignent localement $2 \mathrm{~m} / \mathrm{s}$ [1]. La géométrie à deux entrées de la Baie de Marennes-Oléron entraîne une forte asymétrie entre le flot et le jusant au niveau de l'embouchure de Maumusson: le prisme tidal de flot est de $40 \%$ inférieur au prisme de jusant.

Les littoraux adjacents à l'embouchure subissent une houle moyenne annuelle de l'ordre $1.5 \mathrm{~m}$, qui peut dépasser $6 \mathrm{~m}$ pendant les fortes tempêtes. La direction préférentielle WNW de ces houles induit une dérive littorale annuelle nette vers le sud de part et d'autre de l'embouchure, estimée à l'aide de formules empiriques à plus de $500000 \mathrm{~m}^{3}$ [1]. 
Ces paramètres hydrodynamiques placent l'embouchure tidale de Maumusson dans la catégorie des embouchures à énergie mixte (houle, marée) définie par Hayes [7], avec une prédominance de la marée.

\section{Méthodes et données:}

\subsection{Données bathymétriques:}

Pour des raisons historiques, plusieurs jeux de données bathymétriques dont la fiabilité a déjà été vérifiée ([2] [3] [4] [5]) sont disponibles sur toute la zone d'étude depuis 1824 (1824, 1882, 1960-1970 et 1995). Les données les plus anciennes proviennent de la base de données du Service Hydrographique et Océanographique de la Marine (S.H.O.M.) et ont été digitalisées sur les minutes originales. Les données les plus récentes proviennent des levés de la cellule hydrographique locale (DDE17). Ces données ont été géoréférencées à l'aide du S.I.G. Arcview . Le système géodésique NTF a été choisi et deux types de coordonnées ont été calculées : coordonnées géographiques pour les illustrations et coordonnées Lambert II pour les exploitations quantitatives et la modélisation hydrodynamique. La fiabilité de ces données et de cette méthodologie a été vérifiée au niveau de plusieurs plateaux rocheux dans la baie; la précision relative verticale de l'ordre de $35 \mathrm{~cm}$ a déjà permis de quantifier précisément des évolutions morphologiques dans la zone d'étude [2] [3] [4] [5].

\subsection{Données courantométriques:}

De nombreux jeux de données courantométriques sont disponibles sur la zone d'étude de 1971 à 1973 et ont été acquises dans le cadre de la thèse de Tesson (1973). Ces données ont été obtenues à l'aide de deux ou trois courantomètres dans la tranche d'eau, placés en différents endroits de la baie pendant un cycle tidal. 6 courantomètres déployés perpendiculairement à l'embouchure de Maumusson ont permis d'estimer le prisme tidal de vives-eaux par interpolation latérale et verticale. Les données disponibles au niveau de l'entrée nord ont été utilisées pour vérifier la validité de la simulation numérique à cette époque.

Deux campagnes de mesure ADCP ont été conduites en 2002 (marnage $5.9 \mathrm{~m}$ ), dans le but de calculer le prisme tidal à Maumusson en 2002 d'une part, et de calibrer la simulation hydrodynamique d'autre part.

\subsection{Modélisation hydrodynamique:}

Le système telemac2D [8] a été utilisé dans le but de simuler la marée sur la zone d'étude pour quatre configurations bathymétriques différentes (1824, 1882, 1960-70, 1995). Il s'agit d'un modèle aux éléments finis, qui résout les équations de Navier-Stokes, moyennées sur la hauteur d'eau. La discrétisation en espace a été réalisée grace à un maillage triangulaire où la taille des mailles varie de $3000 \mathrm{~m}$ au large à $50 \mathrm{~m}$ au niveau du pertuis de Maumusson. Le domaine modélisé s'étend de l'estuaire de la Gironde vers le sud, au nord de l'île de Ré vers le nord tandis que la limite au large décrit un arc de cercle de $60 \mathrm{~km}$ autour de l'île d'Oléron. Les forçages du modèle au niveau des conditions aux limites incluent : (1) La marée, calculée à chaque pas de temps à partir d'un série de 30 points disposés le long de la frontière ouverte et pour lesquels les amplitudes et phases des 17 harmoniques principales de la marée ont été prises en compte; (2) les débits des fleuves Charente et Gironde, pour lesquels les valeurs annuelles moyennes ont été considérées. 

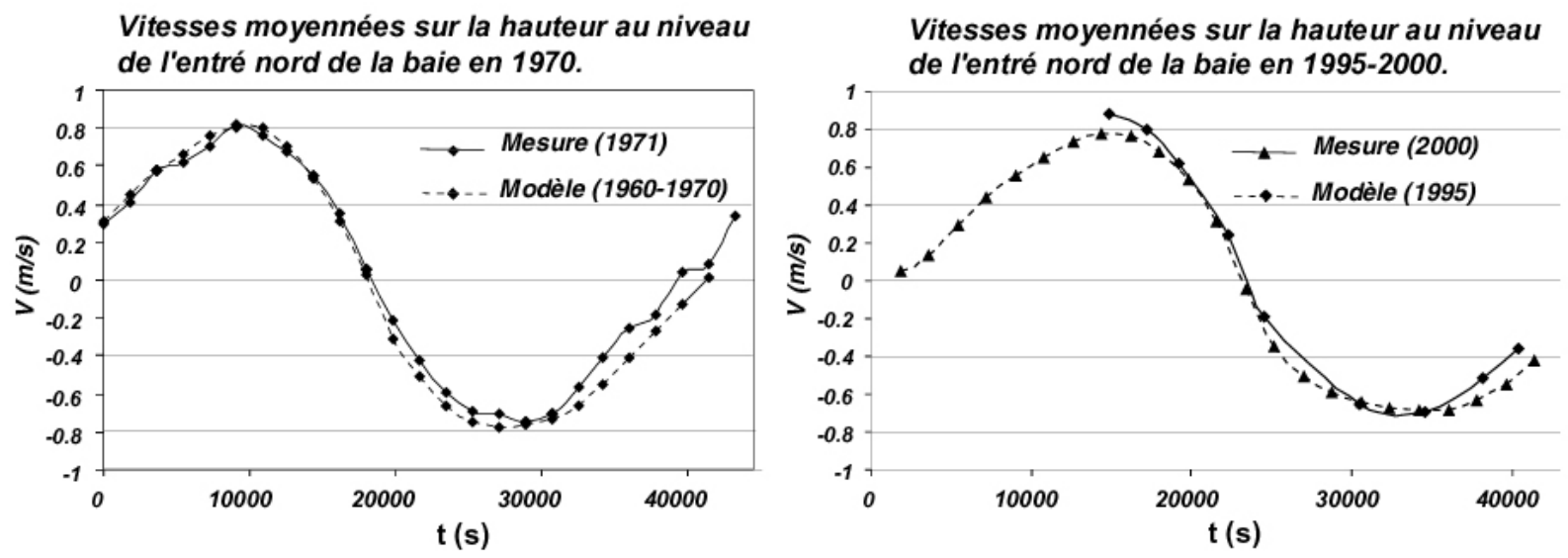

Figure 2. Superposition des données in situ et des résultats prédits par le modèle en 1970 et 1995.Pour ces deux dates, le modèle reproduit aussi bien les intensités de courant que les hauteurs

La calibration de la simulation de 1995 a été effectuée à l'aide des hauteurs d'eau du port de La Rochelle et des données ADCP à l'entrée nord de la baie, moyennées sur la hauteur d'eau (Figure 2). Les simulations de 1960-70, 1882 et 1824 ont été lancées avec les mêmes conditions à la limite, les mêmes conditions initiales et les mêmes paramètres physiques et numériques. La simulation de 1960-70 reproduisant parfaitement les données de terrain de cette même époque (Figure 2), on peut émettre l'hypothèse que cette paramétrisation soit convenable pour les simulations de 1882 et 1824, où les mesures in situ font évidemment défaut.

\section{Evolutions morphologiques et hydrodynamiques de la zone d'étude :}

\subsection{Evolution de la section de l'embouchure de Maumusson:}

Neuf jeux de données bathymétriques disponibles au niveau de l'embouchure de Maumusson ont permis d'étudier les variations de sa section mouillée minimale depuis 1824 . On peut distinguer trois étapes principales dans ces évolutions [2] [3] :

-De 1824 à 1882, la section présente une certaine stabilité et évolue autour de $10000 \mathrm{~m}^{2}$.

- De 1882 à 1970, la section diminue sensiblement jusqu'à $8000 \mathrm{~m}^{2}$.

- De 1970 à 2001, cette tendance semble s'accélérer, la section minimale de l'embouchure n'est plus que de $6800 \mathrm{~m}^{2}$ en 2001.

La diminution de section mouillée de l'embouchure depuis 1824 est de l'ordre de 35\%, traduisant son comblement général.

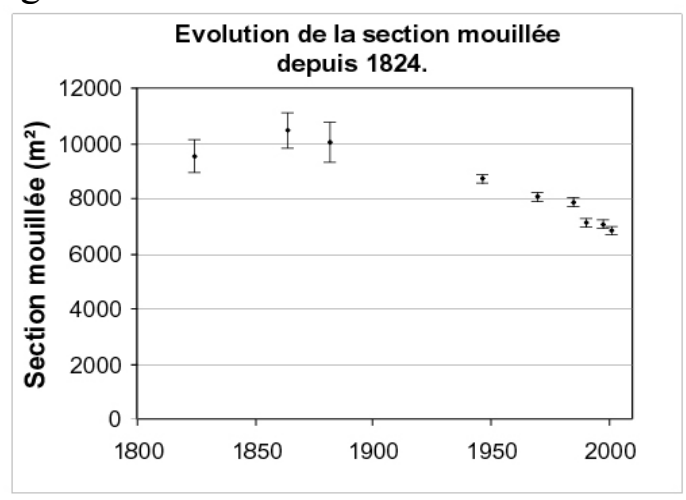

Figure 3. Evolution de la section mouillée minimale de l'embouchure de Maumusson depuis 1824. 


\subsection{Evolutions morphologiques de la Baie tidale:}
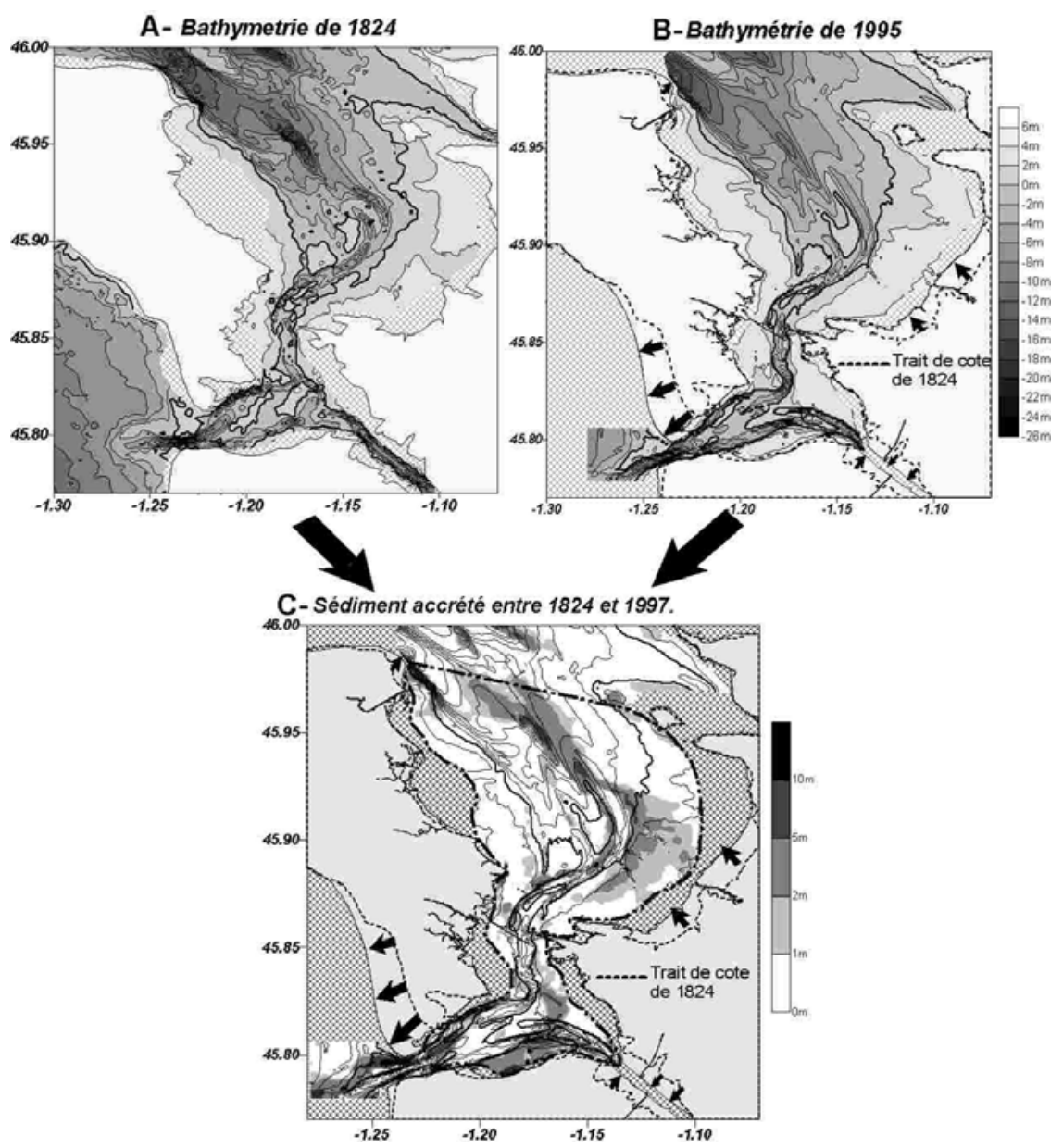

Figure 4 . Evolutions morphologiques dans la baie de MarennesOléron depuis 1824, mettant en évidence de fortes zones en accrétion.

La carte différentielle entre les bathymétries de 1824 et 1995 (figure 4) montre clairement des zones de forts gains sédimentaires dans la baie de Marennes-Oléron. Ces zones se situent principalement :

- au niveau des connections de la baie avec l'océan ouvert.

- dans la partie inférieure des estrans.

- au niveau du large chenal à l'entrée nord de la baie où l'accrétion verticale atteint localement $10 \mathrm{~m}$.

Le volume accrété durant cette période est de $106.10^{6} \mathrm{~m}^{3}$ et entraîne une diminution du volume de remplissage de la baie de l'ordre de $20 \%$.

\subsection{Evolutions des débits tidaux :}

O'Brien [9] [10] fut le premier à proposer une relation reliant le prisme tidal $(\Omega)$ à la section mouillée (A) d'une embouchure tidale. Cette relation est de la forme:

\section{$\mathrm{A}=\mathrm{C} . \Omega^{\mathrm{n}}$}

Où $\mathrm{c}$ et $\mathrm{n}$ sont des constantes déterminées par régression linéaire à partir de bases de données contenant un grand nombre d'embouchures. 
Connaissant (A) et $(\Omega)$ en 2002 , on peut exprimer le prisme tidal à l'instant t $\left(\Omega_{\mathrm{t}}\right)$ :

$$
\Omega_{t}=\frac{\Omega_{2002}}{\sqrt[n]{\frac{A_{2002}}{A_{t}}}}
$$
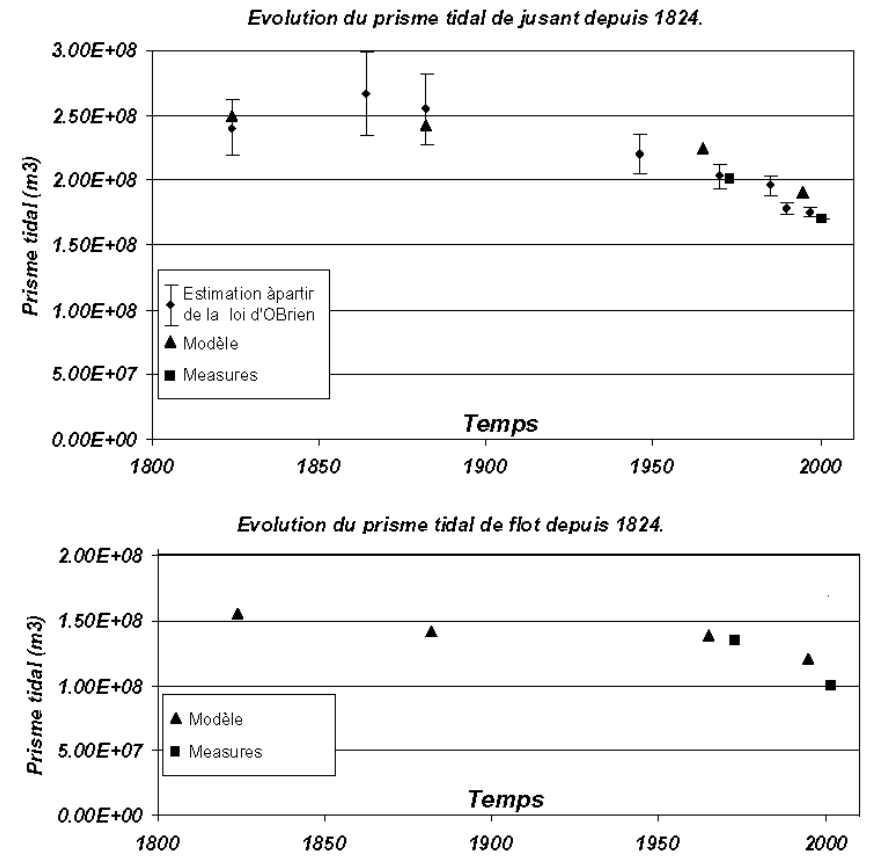

Figure 5.Evolution des prisme tidaux de flot et de jusant à l'embouchure de Maumusson depuis 1824, à partir des variations de section mouillée, des simulations hydrodynamiques et de mesures de courant.

La figure 5 superpose les variations de prisme tidal déduites de cette relation $(1824,1864$, $1882,1946,1970,1985,1990,1997$ et 2001) les prismes tidaux calculés à partir des simulations hydrodynamiques $(1824,1882,1960$ et 1995) et ceux calculés à partir de mesures de courant $(1970,2001)$. Les résultats de ces trois méthodes indépendantes montrent la même tendance, à savoir une diminution des prismes tidaux de flot et jusant de l'ordre de $40 \%$ depuis 1824, avec une accélération depuis 1970.

\section{Discussion et conclusion:}

Les évolutions morphologiques de la baie de Marennes-Oléron mettent en évidence un budget sédimentaire nettement positif depuis 1824. Les différentes zones de forte accrétion sédimentaire ont entraîné une diminution de son volume de remplissage en eau de l'ordre de $20 \%$. Les prismes tidaux étant fonction de ce volume de remplissage, le comblement de la baie peut expliquer de façon directe une partie de la diminution des prismes tidaux au niveau de l'embouchure de Maumusson.

En raison de sa morphologie particulière à deux entrées, la relation entre les débits tidaux et le volume de remplissage de la baie n'est pas linéaire. La forte asymétrie entre les prismes de flot et le jusant illustre bien ce propos. Dès lors, les prismes tidaux à Maumusson sont aussi fonction de la propagation de l'onde de marée dans la Baie de Marennes-Oléron. La diminution de la profondeur au niveau des deux embouchures et la diminution globale des fonds dans la baie peuvent donc être responsables d'une augmentation des frottements concernant la propagation de la marée, ayant pour conséquence une diminution des débits tidaux. Pour expliquer que ces évolutions semblent s'accélérer dans le temps, on peut postuler 
l'existence d'une boucle de rétroaction positive, où la sédimentation dans la baie entraîne une diminution des débits tidaux, diminution entraînant elle-même une augmentation de la sédimentation.

Comme le montre la relation d'O'Brien [9] [10], la section mouillée d'une embouchure tidale est controlée par son prisme tidal. La diminution du prisme tidal à Maumusson, de l'ordre de $40 \%$ depuis 1824 , permet donc d'expliquer facilement la diminution de la section $\mathrm{du}$ chenal et son comblement depuis cette date. Le fonctionnement général d'une embouchure tidale et de ses littoraux adjacents étant gouverné à la fois par la dérive littorale et par le prisme tidal, la diminution de ce dernier, en réponse au comblement de la baie entraîne un bouleversement du système. Dans le cas du Pertuis de Maumusson, la diminution importante des débits tidaux depuis 1970 a entraîné la migration et le basculement du chenal vers le sud, entraînant à leur tour une érosion très forte du littoral amont à la dérive littorale [2] [3].

La valorisation de données bathymétriques séculaires permet dans cette étude de quantifier les évolutions morphologiques et hydrodynamiques à hyper-échelle d'un système baieembouchure tidale, mettant en évidence la réponse de l'embouchure au comblement sédimentaire de la baie.

\section{Références:}

1.Baxerres, P.,1978. Etude morphologique et sédimentologique de la cote atlantique de la pointe sud d'Oléron a la pointe de la Coubre (France). Unpublished P.H.D. Thesis, University of Bordeaux I, 190p.

2.Bertin, X., Chaumillon, E., Weber, N. and Tesson, M., 2003. Morphodynamical evolution and time-varying bedrock control of main channel at a mixed energy tidal inlet: The Maumusson Inlet, France. Marine Geology, in press.

3.Bertin X., Chaumillon E., Weber N. and Tesson M., 2003. Morphological evolution and coupling with bedrock substratum at a mixed energy tidal inlet: The Maumusson inlet, France. In: Sanchez-Arcilla A and Bateman A (Ed), proceedings of the 3rd IAHRD symposium on river, coastal and estuarine morphodynamics (RCEM 2003) IAHRD publication, Madrid, Spain: 494-503.

4.Chaumillon E., Bertin X., Weber N. and Tesson M., 2003. Internal architecture and sedimentary facies of a macrotidal bay: implication in terms of morphodynamics (The Marenne-Oléron bay, Atlantic coast of France). In: Sanchez-Arcilla A and Bateman A (Ed), proceedings of the 3rd IAHRD symposium on river, coastal and estuarine morphodynamics (RCEM 2003) IAHRD publication, Madrid, Spain: 423-432.

5.Chaumillon, E., Gillet, H., Weber, N., Walker, P. And Tesson, M., 2002. Evolution temporelle et architecture interne d'un banc sableux estuarien : la Longe de Boyard (littoral Atlantique, France). C.R. Geosciences, 334, 119-126.

6.FitzGerald, D.M.,1996. Geomorphologic variability and morphodynamic and sedimentologic controls on tidal inlets. Journal of coastal research, vol S.I., $N^{\circ} 23,47-71$.

7.Hayes, M.O.,1979. Barrier island morphology as a function of tidal and wave regime. Barrier island, S.P. Leatherman edition, Academic press, New York, 1-28.

8.Hervouet, J.M., Van Haren, L., 1994. Telemac 2-D Version 3.0 Principle Note, EDFDER report HE-43/94/052/B.

9.0’Brien, M.P., 1931. Estuary tidal prism related to entrance area . Civil Eng., 1:738739.

10.O'Brien, M.P., 1969. Equilibrium flow areas of inlets on sandy coasts. Journal of Waterways and Harbours Division, 13th Coastal Engr. Conf., p.761-780.

11.Tesson, M., 1973. Aspects dynamiques de la sédimentation dans la baie de Marennes Oléron (Charente-Maritime). Unpublished P.H.D. Thesis, University of Bordeaux, 128p. 